\title{
Prenatal exposure to organochlorine pesticides and TSH status in newborns from Southern Spain
}

\author{
Carmen Freire ${ }^{\text {a,* }}$, Maria-Jose Lopez-Espinosa ${ }^{\text {a,b }}$, Marieta Fernández ${ }^{\text {a }}$, Jose-Manuel Molina-Molina ${ }^{\text {a }}$, \\ Remedios Prada ${ }^{a}$, Nicolas Olea ${ }^{\text {a }}$
}

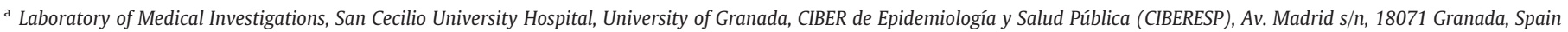

${ }^{b}$ Department of Social and Environmental Health Research, London School of Hygiene and Tropical Medicine, 15-17 Tavistock Place, WC1H 9SH London, UK

\section{A R T I C L E I N F O}

\section{Article history:}

Received 14 December 2010

Received in revised form 10 May 2011

Accepted 18 May 2011

\section{Keywords:}

Prenatal exposure

Male birth cohort

Organochlorine pesticides (OCPs)

Thyroid-stimulating hormone (TSH)

Endocrine disruption

\begin{abstract}
A B S T R A C T
Objective: To investigate the association between prenatal exposure to organochlorine pesticides (OCPs) and thyroid-stimulating hormone (TSH) levels in male newborns.

Methods: Exposure to 17 OCPs was analyzed in 220 placentas from a male birth cohort in Southern Spain, and TSH was measured in the umbilical cord blood. OCP concentrations were quantified by gas chromatography and mass spectrometry. Multivariate regression analysis was conducted to examine the association between pesticide exposure and neonatal TSH levels, adjusting for confounders.

Results: Newborn boys with higher exposure to endrin in placenta had higher odds of TSH cord blood levels $\geq 5 \mathrm{mU} / \mathrm{L}$ (80th percentile) ( $\mathrm{OR}=2.05 ; 95 \% \mathrm{CI}=1.01,4.18 ; p=0.05)$, whereas higher prenatal exposure to endosulfan-sulfate was associated with lower odds of TSH $\geq 5 \mathrm{mU} / \mathrm{L}$ (OR=0.36; $95 \% \mathrm{CI}=0.17,0.77$; $p=0.008)$. A marginally significant negative association was found between TSH and hexachlorobenzene levels $(\beta=-0.15 ; 95 \% \mathrm{CI}=-0.31,0.02 ; p=0.09)$, and exposure to $p, p^{\prime}$-DDE showed a marginally-significant higher odds of TSH $\geq 5 \mathrm{mU} / \mathrm{L}(\mathrm{OR}=1.32 ; 95 \% \mathrm{CI}=0.95,1.83 ; p=0.09)$. No association was found between TSH and the remaining pesticides.

Conclusions: Early exposure to certain environmental chemicals with endocrine-disruption activity may interfere with neonatal thyroid hormone status; however, the pattern of interference is not yet clearly elucidated.
\end{abstract}

(C) 2011 Elsevier B.V. All rights reserved.

\section{Introduction}

Human exposure to environmental chemicals that disrupt endocrine homeostasis (endocrine disruptors [EDs]) has been mainly related to effects on sex hormones (estrogens and androgens). Thus, many organochlorine compounds (OCs) have been shown to display an estrogenic-like activity (DeCastro et al., 2006; Fernández et al., 2004). Organochlorine pesticides (OCPs), polychlorinated biphenyls (PCBs) and dioxins/furans have also been demonstrated to influence thyroid hormone $(\mathrm{TH})$ (thyroxine $\left[\mathrm{T}_{4}\right]$, triiodothyronine $\left[\mathrm{T}_{3}\right]$ and thyroidstimulating hormone [TSH]) activity and metabolism in animal studies (Boas et al., 2006). With regard to OCPs, there are considerable animal data on the alteration of TH levels by exposure to $p, p^{\prime}$-dichlorodiphenyldichloroethene ( $p, p^{\prime}$-DDE) and hexachlorobenzene (HCB) (ATSDR, 2002a, 2002b).

Abbreviations: OCPs, organochlorine pesticides; TSH, thyroid-stimulating hormone; $\mathrm{ED}$, endocrine disruptor; OC, organochlorine compound; TH, thyroid hormone.

* Corresponding author. Laboratory of Medical Investigations, San Cecilio University Hospital, University of Granada, Av. Madrid s/n, 18071 Granada, Spain. Tel.: +34 958240758; fax: + 34958249953.

E-mail addresses: cfreire@ugr.es (C. Freire),mjlopes@ugr.es (M.-J. Lopez-Espinosa), marieta@ugr.es (M. Fernández), molinajm@ugr.es (J.-M. Molina-Molina), reprama@hotmail.com (R. Prada),nolea@ugr.es (N. Olea).
Thyroid disruption may be caused by various mechanisms, since different chemicals interfere with the hypothalamic-pituitary-thyroid axis at distinct levels. Several OCs are structurally similar to THs and therefore have the potential to bind to proteins and receptors that characteristically bind THs, producing complex effects on TH signaling (Zoeller, 2007). In addition, changes in serum TH concentrations can be caused by chemicals that inhibit TH synthesis, release and metabolism (Boas et al., 2006).

Several studies have investigated associations between OC exposure levels in human tissues and TH status in adults (Langer et al., 2006) and infants (Alvarez-Pedrerol et al., 2008; Asawasinsopon et al., 2006; Lopez-Espinosa et al., 2010; Ribas-Fitó et al., 2003; Takser et al., 2005), with no conclusive results (Boas et al., 2006; Maervoet et al., 2007). Discrepancies in these findings might result from differences in study populations, sample sizes, matrices for exposure measurement (e.g., maternal venous blood, cord blood, breast milk), specimen collection time points and/or isomers. Investigations into the effects of early OC exposure on human thyroid function have largely studied PCBs and dioxins (Maervoet et al., 2007; Wang et al., 2005). Recent studies suggested that $p, p^{\prime}$-DDT (1,1,1-trichloro-2,2-bis(p-chlorophenyl) ethane), $p, p^{\prime}$-DDE, $\beta$-hexachlorocyclohexane $(\beta-\mathrm{HCH})$ and $\mathrm{HCB}$ may also alter the thyroid system (Alvarez-Pedrerol et al., 2008, 2009a, 2009b; Takser et al., 2005). However, little is known about the disrupting 
potential of OCPs other than DDT, $\mathrm{HCB}$, or $\mathrm{HCH}$, e.g., mirex, aldrin and dieldrin (Asawasinsopon et al., 2006; Takser et al., 2005).

THs play an important role during early human development and it has been speculated that some of the neurotoxic and reproductive effects of early exposure to environmental EDs may result from disruption of the thyroid system (Williams, 2008). Thus, studies in infants have focused on the association between THs and adverse health effects such as low birth weight, growth retardation and altered psychomotor and cognitive functions (Alvarez-Pedrerol et al., 2009a, 2009b; Freire et al., 2010b; Ribas-Fitó et al., 2007; Toft et al., 2004).

The production and use of most OCPs have been restricted or banned internationally since the 1970s, although some remain in legal use in various countries (Beard, 2006; PNA Convenio Estocolmo y Reglamento 850/2004., 2007). However, worldwide and intensive use of these compounds has led to widespread contamination of the environment and, due to their high persistence and bioaccumulation capacity, their presence is still detected in human tissues (Cerrillo et al., 2005; Lopez-Espinosa et al., 2007). Southern Spain has the largest area of intensive greenhouse agriculture in Europe and may constitute a special case of exposure to pesticides that have only recently been banned (in 2009), such as endosulfans (Cerrillo et al., 2005). In fact, Spain has been the main consumer of endosulfans in the European Union over the past few years (Endosulfan Preliminary Dossier, 2003).

Exposure to OCPs during human development may result in a permanent alteration of the endocrine system, and the fetus can be exposed to these compounds through the placenta. The present study aimed to investigate the relationship between cord blood TSH levels in a cohort of newborns from Granada, Southern Spain, and their prenatal exposure to a range of OCPs in placentas. This investigation is part of the INMA (INfancia y Medio Ambiente [Environment and Childhood]) Project, a prospective multi-center study in Spain (www. proyectoinma.org).

\section{Methods}

\subsection{Subject recruitment}

From 2000 to 2002, 700 eligible mother-son pairs registered at the San Cecilio University Hospital were enrolled at delivery, establishing the INMA-Granada male birth cohort, with the initial aim of investigating the relationship between chronic exposure to endocrine disrupting pesticides during pregnancy and urogenital malformations in newborn boys (Freire et al., 2010a; Lopez-Espinosa et al., 2007). Exclusion criteria were: maternal presence of serious chronic disease, such as diabetes, hypertension or thyroid disease; pregnancy complication that could affect growth or development; and non-residence in the hospital referral area (Fernández et al., 2007). The study was approved by the Institutional Ethical Committee of the Hospital, and signed informed consent was obtained from the eligible women who agreed to participate.

\subsection{Laboratory analysis}

\subsubsection{Extraction and quantification of OCPS}

A random sample of 308 placentas was selected from the cohort for chemical analysis. Placentas were collected at delivery and sent to the Laboratory of Medical Investigations for analysis. They were immediately coded, frozen, and stored at $-86{ }^{\circ} \mathrm{C}$ until processing (Lopez-Espinosa et al., 2007). Before analysis, half of the placenta was almost defrosted and then mechanically homogenized. Bioaccumulated compounds were extracted from samples by a previously described method (Fernández et al., 2007) with slight modifications. Briefly, $1.6 \mathrm{~g}$ of placenta homogenate was dissolved in hexane and eluted in a glass column filled with Alumine (Merck, Darmstadt, Germany). The eluate obtained was concentrated at reduced pressure under nitrogen stream and then injected into the preparative high pressure liquid chromatography (HPLC). This previously described HPLC method allows us the separation of xenoestrogens from natural estrogens without destroying them. In addition, it has been demonstrated that the fraction collected in the first 11 min HPLC ( $\alpha$ fraction) contains all the selected persistent chemicals (Fernández et al., 2007).

The presence of 17 OCPs (o, $p^{\prime}$-DDT, $p, p^{\prime}$-DDT, $o, p^{\prime}$-DDD (dichlorodiphenyldichloroethane), $p, p^{\prime}$-DDE, endosulfan-I and -II, endosulfandiol, -sulfate, -lactone, and -ether, aldrin, dieldrin, endrin, lindane, $\mathrm{HCB}$, mirex, and methoxychlor) was determined by gas chromatography with electron-capture detection (Varian-3350 gas chromatograph plus ECD (63Ni)), using $p^{\prime}$-dichlorobenzophenone as internal standard, and mass spectrometry. Standard solutions of organochlorine compounds were analyzed previously to determine retention times and calibration curves of these chemicals. The calibration linearity of all chemicals in pure and processed standards was $>0.98$. The recovery of studied chemicals, measured by spiking placenta samples with pure chemicals, ranged from 84 to $102 \%$. The reproducibility of the process was established by running 10 placenta samples 10 times. The limits of quantification (LQ) of the gas chromatograph for the studied chemicals ranged from 0.1 to $3.0 \mathrm{ng} / \mathrm{mL}$. Chemical compounds were confirmed by GC and mass spectrometry (GC/MS) using a Saturn 2000 ion-trap mass spectrometer from Varian (Fernández et al., 2007; Lopez-Espinosa et al., 2007) in all the samples. Placental lipid content was gravimetrically quantified.

\subsubsection{TSH determination}

TSH was measured in a cord blood sample spotted on a filter paper (Schleicher \& Schuell no. 2992), which was routinely obtained shortly after birth in our Hospital for the national neonatal hypothyroidism screening program (Cortés Castell et al., 2002). Data on neonatal TSH levels were gathered for $80 \%$ of the boys $(n=551)$. Hormone concentrations were determined by using time-resolved sandwich fluoroimmunoassay (AutoDELFIA, Perkin Elmer/Wallac, Turku, Finland) at the Center for the Early Detection of Metabolopathies in Neonates in San Juan de Dios Hospital (Granada, Spain). The limit of detection (LD) was $0.01 \mathrm{mU} / \mathrm{L}$. A cord blood level $\geq 14 \mathrm{mU} / \mathrm{L}$ is established in the Center's laboratory to trigger the protocol for the study and confirmation of neonatal hypothyroidism.

\subsection{Covariates}

Covariates considered in the statistical analysis are shown in Table 1 . A post-delivery questionnaire was administered to gather data on: maternal age, alcohol consumption and cigarette smoking during pregnancy, parity, pre-pregnancy body mass index (BMI), maternal education, marital status, and area of residence (urban: city of Granada; metropolitan: towns of $>20,000$ inhabitants in city residential belt; sub-urban: towns of $10,000-20,000$ inhabitants; rural: $<10,000$ inhabitants), among other reproductive and medical characteristics. Information on gestational age and anthropometric measurements at birth were obtained from medical records. We considered these covariates as potential confounders a priori.

Complete information on placental OCP concentrations, cord blood TSH levels and covariates were available for a subset of 220 subjects from the cohort. No differences in any study characteristics were found between this subset and the children without TSH measurements $(n=88)$ (data not shown).

\subsection{Statistical analysis}

Descriptive analysis of continuous OCP concentrations was first conducted. For further analyses, values of those OCPs with a frequency of detection $\geq 50 \%$ were transformed into natural logarithms and treated as continuous variables (for levels below the LD, we considered a value of half the $\mathrm{LD}$ ); while concentrations of pesticides 
Table 1

TSH levels $(\mathrm{mU} / \mathrm{L})$ measured in cord blood $(\mathrm{n}=220)$ by characteristics of study population from the INMA-Granada cohort, Southern Spain, $2000-2002$.

\begin{tabular}{|c|c|c|c|c|}
\hline & \multirow[t]{2}{*}{$\mathrm{n}(\%)$} & \multicolumn{3}{|c|}{ TSH levels (mU/L) } \\
\hline & & GM & $95 \% \mathrm{CI}$ & $\overline{p-\text { value }^{\mathrm{a}}}$ \\
\hline \multicolumn{5}{|l|}{ Child variables } \\
\hline Small for gestational age & & & & 0.72 \\
\hline No & $197(89.5)$ & 3.05 & $2.79 ; 3.34$ & \\
\hline Yes & $22(10.5)$ & 3.21 & $2.51 ; 4.11$ & \\
\hline Gestational age (weeks) ${ }^{\mathrm{b}}$ & $220(100)$ & 39.4 & $39.2 ; 39.6$ & 0.76 \\
\hline Birth weight $(\mathrm{g})^{\mathrm{b}}$ & $220(100)$ & 3335 & $3277 ; 3394$ & 0.26 \\
\hline \multicolumn{5}{|l|}{ Maternal variables } \\
\hline Age at delivery (years) ${ }^{\mathrm{b}}$ & $220(100)$ & 31.8 & $31.2 ; 32.5$ & 0.44 \\
\hline Smoking during pregnancy & & & & 0.16 \\
\hline No & $168(76.4)$ & 3.17 & $2.89 ; 3.48$ & \\
\hline Yes & $52(23.6)$ & 2.76 & $2.29 ; 3.33$ & \\
\hline Alcohol consumption & & & & 0.12 \\
\hline No & $196(89.1)$ & 3.15 & $2.90 ; 3.42$ & \\
\hline Yes & $20(10.9)$ & 2.52 & $1.71 ; 3.71$ & \\
\hline Parity & & & & 0.14 \\
\hline Primiparous & $110(50)$ & 3.27 & $2.91 ; 3.67$ & \\
\hline Multiparous & $110(50)$ & 2.88 & $2.56 ; 3.25$ & \\
\hline Pre-pregnancy BMI $\left(\mathrm{kg} / \mathrm{m}^{2}\right)$ & & & & 0.92 \\
\hline$<19$ & $13(5.9)$ & 3.20 & $2.41 ; 4.26$ & \\
\hline $19-24$ & $142(64.5)$ & 3.06 & $2.74 ; 3.42$ & \\
\hline $25-29$ & $51(23.2)$ & 3.07 & $2.66 ; 3.55$ & \\
\hline$\geq 30$ & $14(6.4)$ & 3.04 & $1.97 ; 4.69$ & \\
\hline Educational level & & & & 0.90 \\
\hline Up to primary school & $115(52.3)$ & 3.05 & $2.70 ; 3.45$ & \\
\hline Secondary school & $69(31.4)$ & 3.03 & $2.60 ; 3.54$ & \\
\hline University & $36(16.4)$ & 3.20 & $2.73 ; 3.76$ & \\
\hline Marital status & & & & 0.80 \\
\hline Stable partner & $202(91.8)$ & 3.08 & $2.82 ; 3.36$ & \\
\hline Without stable partner & $18(8.2)$ & 2.97 & $2.32 ; 3.79$ & \\
\hline Area of residence & & & & 0.51 \\
\hline Rural & $50(22.7)$ & 2.81 & $2.28 ; 3.45$ & \\
\hline Sub-urban & $35(15.9)$ & 3.45 & $2.93 ; 4.06$ & \\
\hline Metropolitan & $108(49.0)$ & 3.10 & $2.75 ; 3.49$ & \\
\hline Urban & $27(12.3)$ & 2.99 & $2.35 ; 3.81$ & \\
\hline
\end{tabular}

TSH: thyroid-stimulating hormone; GM: geometric mean; CI: confidence interval.

a $\mathrm{p}$-value for simple linear regression, $T$-test or ANOVA.

b Continuous variables; BMI: body mass index.

detected in $<50 \%$ of the placenta extracts were treated as dichotomous variables, using the LD as cut-off. TSH values were transformed into natural logarithms to improve the normality and were treated as a continuous variable. In a second analysis, TSH was dichotomized using as cutoff the value established by the World Health Organization for "moderately elevated neonatal concentrations" (5 mU/L) (WHO, 2007), which coincided with the 80th percentile (4.99 mU/L). We used one-way analysis of variance and the Student's $t$-test to examine the relationship of covariates with continuous TSH levels.

Linear and logistic regression analyses were performed to explore the unadjusted and adjusted association of OCP concentrations in placenta tissue samples with cord blood TSH levels. All multivariate models controlled for maternal age and gestational age (continuous), regardless of their statistical significance, based on reports in the literature (Chevrier et al., 2008; Maervoet et al., 2007; Takser et al., 2005). OCP concentrations were adjusted by g of placenta ( $\mathrm{ng} / \mathrm{g}$ ) in the descriptive analysis, but the total placental lipid content was included as a separate term in the regression models for continuous OCPs because it was recently shown that lipid standardization models are highly prone to bias (Schisterman et al., 2005). Models were also adjusted for confounding factors, i.e., prenatal smoking (yes/no), alcohol consumption during pregnancy (yes/no), parity (primiparous/ multiparous), birth weight (continuous) and maternal education (primary/secondary/university). Variables associated with TSH levels at a significance level of $p<0.20$ in bivariate analysis or whose inclusion in the models changed OCP effect estimates by $>10 \%$ were
Table 2

Organochlorine pesticide (OCP) concentrations measured in placenta (ng/g placenta), INMA-Granada cohort, Southern Spain, 2000-2002 $(\mathrm{n}=220)$.

\begin{tabular}{|c|c|c|c|c|c|c|c|c|}
\hline OCPs & $\mathrm{n}(\% \geq \mathrm{LD})$ & $\mathrm{GM}^{\mathrm{a}}$ & $95 \% \mathrm{Cl}^{\mathrm{a}}$ & P25 & P50 & P75 & P95 & Max. \\
\hline$o, p^{\prime}-\mathrm{DDT}$ & 123 (55.9) & 0.86 & $0.76 ; 0.97$ & $<\mathrm{LD}$ & 0.50 & 0.73 & 3.18 & 6.98 \\
\hline$p, p^{\prime}-\mathrm{DDT}$ & $108(49.1)$ & 1.25 & $1.03 ; 1.52$ & $<\mathrm{LD}$ & $<\mathrm{LD}$ & 0.91 & 7.47 & 51.2 \\
\hline$p, p^{\prime}-\mathrm{DDE}$ & 205 (93.2) & 2.01 & $1.75 ; 2.30$ & 0.84 & 1.74 & 3.37 & 11.9 & 65.7 \\
\hline$o, p^{\prime}-\mathrm{DDD}$ & $108(49.1)$ & 1.91 & $1.54 ; 2.37$ & $<\mathrm{LD}$ & $<\mathrm{LD}$ & 1.86 & 15.1 & 35.3 \\
\hline$\sum$ DDTs & $215(97.7)$ & 4.16 & $3.61 ; 4.81$ & 1.99 & 4.13 & 7.89 & 25.5 & 66.5 \\
\hline E-I & $116(52.7)$ & 0.73 & $0.60 ; 0.88$ & $<\mathrm{LD}$ & 0.25 & 0.58 & 5.11 & 29.8 \\
\hline E-II & 69 (31.4) & 1.37 & $1.14 ; 1.64$ & $<\mathrm{LD}$ & $<\mathrm{LD}$ & 1.00 & 10.8 & 48.7 \\
\hline E-diol & $116(63.2)$ & 2.10 & $1.07 ; 6.20$ & $<\mathrm{LD}$ & 3.68 & 6.27 & 18.7 & 26.2 \\
\hline E-ether & $110(50.0)$ & 0.23 & $0.20 ; 0.27$ & $<\mathrm{LD}$ & $<\mathrm{LD}$ & 0.19 & 1.36 & 4.67 \\
\hline E-sulfate & 109 (49.5) & 0.93 & $0.75 ; 1.14$ & $<\mathrm{LD}$ & $<\mathrm{LD}$ & 0.88 & 7.17 & 31.7 \\
\hline E-lactone & 85 (38.6) & 1.14 & $0.87 ; 1.50$ & $<\mathrm{LD}$ & $<\mathrm{LD}$ & 0.79 & 17.2 & 40.3 \\
\hline$\sum$ Endosulfans & 211 (95.9) & 4.02 & $3.35 ; 4.82$ & 1.26 & 5.69 & 9.64 & 27.0 & 129 \\
\hline Aldrin & $62(28.2)$ & 0.82 & $0.68 ; 0.99$ & $<\mathrm{LD}$ & $<\mathrm{LD}$ & 0.50 & 4.41 & 7.86 \\
\hline Endrin & $75(34.1)$ & 2.53 & $2.12 ; 3.01$ & $<\mathrm{LD}$ & $<\mathrm{LD}$ & 1.50 & 10.8 & 59.1 \\
\hline Dieldrin & 47 (21.4) & 1.05 & $0.82 ; 1.34$ & $<\mathrm{LD}$ & $<\mathrm{LD}$ & $<\mathrm{LD}$ & 8.73 & 10.0 \\
\hline Lindane & $168(76.4)$ & 0.41 & $0.32 ; 0.53$ & 0.12 & 0.25 & 0.47 & 1.84 & 6.66 \\
\hline $\mathrm{HCB}$ & $94(42.7)$ & 1.02 & $0.84 ; 1.24$ & $<\mathrm{LD}$ & $<\mathrm{LD}$ & 0.55 & 8.62 & 30.4 \\
\hline Methoxychlor & $62(28.2)$ & 1.20 & $0.94 ; 1.52$ & $<\mathrm{LD}$ & $<\mathrm{LD}$ & 0.50 & 10.0 & 22.0 \\
\hline Mirex & $55(25.0)$ & 1.15 & $0.91 ; 1.47$ & $<\mathrm{LD}$ & $<\mathrm{LD}$ & 0.44 & 8.72 & 22.3 \\
\hline
\end{tabular}

LD: Limit of detection; GM: geometric mean; CI: confidence interval; P: percentile; Max.: maximum.

DDT: 1,1,1-tricloro-2,2-bis(4-clorofenil)-etano; $\sum$ DDTs: sum of o, $p^{\prime}$-DDT, $p, p^{\prime}$-DDT, $p, p^{\prime}$ DDE, and $o, p^{\prime}$-DDD; E: endosulphan; $\sum$ Endosulfans: sum of E-I, E-II, E-diol, E-ether, E-sulfate, and E-lactone; HCB: hexachlorobenzene.

a Descriptive statistics for concentrations $\geq$ LD. 
considered confounders. $p \leq 0.05$ was considered significant. SPSS version 16.0 (SPSS Inc., Chicago, IL, US) and STATA version 9.0 (Corporation, College Station, Texas) were used for the analyses.

\section{Results}

The mean age of the 220 mothers at delivery was 32 years (range $=16-46$ years) and pre-pregnancy BMI was $24 \mathrm{~kg} / \mathrm{m}^{2}$ (17$40 \mathrm{~kg} / \mathrm{m}^{2}$ ). Around $24 \%$ of women were smokers, $9 \%$ consumed alcohol during pregnancy, 50\% were primiparous, $16 \%$ had university education, and only $8 \%$ did not have a stable partner. The mean birth weight was $3335 \mathrm{~g}$ (2070-4590 g), and the mean duration of gestation was 39 weeks (32-42 weeks); $10 \%$ of the boys were small for gestational age (SGA). All cord blood TSH levels were within normal laboratory reference values $(<14 \mathrm{mU} / \mathrm{L})$, with a geometric mean $(\mathrm{GM})$ of $3.07 \mathrm{mU} / \mathrm{L}$ (95\% confidence interval $[\mathrm{CI}]=2.82,3.34$; range $=0.24-14.00 \mathrm{mU} / \mathrm{L}$ ). Table 1 summarizes TSH levels by characteristics of the study population. Although differences did not reach statistical significance, cord blood TSH levels were higher among primiparous mothers $(p=0.14)$ and those smoking $(p=0.16)$ or consuming alcohol during pregnancy $(p=0.12)$.

Table 2 lists the percentages of detection, and GM, 95\% CI, percentiles 25, 5075 and 95, and maximum levels $\geq$ LD of the 17 OCPs quantified in the placenta samples. All samples were positive for at least one pesticide, with a mean of eight pesticides per placenta (range $=2-15$ pesticides). $p, p^{\prime}$-DDE was the most frequent pesticide (96.2\%), followed by lindane (76.4\%), endosulfan-diol (63.2\%), o, $p^{\prime}-$ DDT (55.9\%), endosulfan-I (52.7\%), and endosulfan-ether (50.0\%). The remaining OCPs studied were detected in $<50 \%(21.4-49.5 \%)$ of placenta samples. Around $98 \%$ and $96 \%$ of the placenta samples, respectively, had detectable levels of at least one of the studied DDT and endosulfan isomers/metabolites. Unadjusted analysis with cate-

Table 3

Unadjusted association of TSH levels ( $\mathrm{mU} / \mathrm{L}$ ) measured in cord blood with organochlorine pesticide (OCP) concentrations (ng/g placenta), INMA-Granada cohort, Southern Spain, 2000-2002 $(n=220)$.

\begin{tabular}{|c|c|c|c|c|c|c|}
\hline \multirow[t]{2}{*}{ OCPs } & \multicolumn{3}{|c|}{$\mathrm{TSH} \geq 5 \mathrm{mU} / \mathrm{L}^{\mathrm{a}}$} & \multicolumn{3}{|c|}{ Continuous $\mathrm{TSH}^{\mathrm{b}}$} \\
\hline & OR & $95 \% \mathrm{CI}$ & p-value & $\beta$ & $95 \% \mathrm{CI}$ & p-value \\
\hline$o, p^{\prime}-\mathrm{DDT}^{\mathrm{c}}$ & 1.02 & $0.67 ; 1.55$ & 0.92 & 0.04 & $-0.06 ; 0.15$ & 0.43 \\
\hline$p, p^{\prime}-\mathrm{DDT}^{\mathrm{d}}$ & 0.83 & $0.43 ; 1.62$ & 0.59 & -0.08 & $-0.25 ; 0.08$ & 0.32 \\
\hline$p, p^{\prime}-\mathrm{DDE}^{\mathrm{C}}$ & 1.35 & $0.99 ; 1.83$ & 0.05 & 0.04 & $-0.03 ; 0.12$ & 0.29 \\
\hline $0, p^{\prime}-\mathrm{DDD}^{\mathrm{d}}$ & 0.93 & $0.48 ; 1.81$ & 0.84 & -0.06 & $-0.23 ; 0.11$ & 0.48 \\
\hline$\Sigma$ DDTs ${ }^{\mathrm{c}}$ & 1.28 & $0.94 ; 1.74$ & 0.11 & 0.00 & $-0.08 ; 0.07$ & 0.91 \\
\hline $\mathrm{E}-\mathrm{I}^{\mathrm{c}}$ & 0.96 & $0.71 ; 1.28$ & 0.77 & -0.03 & $-0.10 ; 0.04$ & 0.45 \\
\hline $\mathrm{E}-\mathrm{II}^{\mathrm{d}}$ & 1.50 & $0.75 ; 2.99$ & 0.25 & 0.01 & $-0.17 ; 0.19$ & 0.91 \\
\hline E-diol ${ }^{\mathrm{C}}$ & 1.17 & $0.92 ; 1.48$ & 0.20 & 0.02 & $-0.04 ; 0.07$ & 0.58 \\
\hline E-ether ${ }^{c}$ & 1.10 & $0.79 ; 1.53$ & 0.58 & 0.00 & $-0.08 ; 0.09$ & 0.94 \\
\hline E-sulfate ${ }^{\mathrm{d}}$ & 0.40 & $0.20 ; 0.80$ & 0.01 & -0.14 & $-0.31 ; 0.02$ & 0.09 \\
\hline E-lactone ${ }^{\mathrm{d}}$ & 0.69 & $0.34 ; 1.39$ & 0.30 & -0.08 & $-0.25 ; 0.09$ & 0.36 \\
\hline ¿Endosulfans ${ }^{c}$ & 1.07 & $0.86 ; 1.35$ & 0.53 & 0.00 & $-0.06 ; 0.05$ & 0.99 \\
\hline Aldrin ${ }^{\mathrm{d}}$ & 1.09 & $0.53 ; 2.25$ & 0.82 & 0.01 & $-0.17 ; 0.20$ & 0.89 \\
\hline Endrin $^{\mathrm{d}}$ & 2.32 & $1.18 ; 4.55$ & 0.01 & 0.08 & $-0.10 ; 0.25$ & 0.39 \\
\hline Dieldrin $^{\mathrm{d}}$ & 1.30 & $0.60 ; 2.81$ & 0.51 & -0.02 & $-0.22 ; 0.18$ & 0.85 \\
\hline Lindane $^{c}$ & 1.01 & $0.67 ; 1.52$ & 0.95 & -0.04 & $-0.14 ; 0.07$ & 0.50 \\
\hline $\mathrm{HCB}^{\mathrm{d}}$ & 0.91 & $0.47 ; 1.78$ & 0.78 & -0.07 & $-0.24 ; 0.10$ & 0.41 \\
\hline Methoxychlor $^{d}$ & 1.61 & $0.80 ; 3.25$ & 0.18 & 0.10 & $-0.08 ; 0.29$ & 0.27 \\
\hline Mirex $^{\mathrm{d}}$ & 1.76 & $0.86 ; 3.60$ & 0.12 & 0.18 & $-0.01 ; 0.37$ & 0.07 \\
\hline
\end{tabular}

OR: Odds ratio; CI: confidence interval; $\beta$ : regression coefficient.

DDT: 1,1,1-trichloro-2,2-bis(4-chlorophenyl)-ethane; ¿DDTs: sum of $o, p^{\prime}$-DDT, $p, p^{\prime}$-DDT, $p, p^{\prime}-\mathrm{DDE}$, and $o, p^{\prime}$-DDD; E: endosulfan; ¿Endosulfans: sum of E-I, E-II, E-diol, E-ether, E-sulfate, and E-lactone; HCB: hexachlorobenzene.

a Simple logistic regression analysis with dichotomous TSH levels ( $\geq 5 \mathrm{mU} / \mathrm{L}$ ) as dependent variable.

b Simple linear regression analysis with log-transformed TSH levels as dependent variable.

c Concentrations transformed into natural logarithm.

d Categorical concentrations ( $\geq$ limit of detection). gorical TSH ( $\geq 5 \mathrm{mU} / \mathrm{L})$ showed positive associations with endrin $(\mathrm{OR}=2.32 ; 95 \% \mathrm{CI}=1.18,4.55 ; p=0.01)$ and $p, p^{\prime}-\mathrm{DDE}(\mathrm{OR}=1.35$; $95 \% \mathrm{CI}=0.99,1.83 ; p=0.05)$ and an inverse relation with endosulfansulfate $(\mathrm{OR}=0.40 ; 95 \% \mathrm{CI}=0.20,0.80 ; p=0.01)$. No significant associations were found with continuous TSH levels (Table 3 ).

Table 4 shows the results of the adjusted analysis of the different OCP concentrations with cord blood TSH levels. Levels of TSH $\geq 5 \mathrm{mU} / \mathrm{L}$ remained significantly associated with endrin and endosulfan-sulfate placental concentrations after controlling for covariates, with a 2-fold higher odds ( $\mathrm{OR}=2.05 ; 95 \% \mathrm{CI}=1.01,4.18 ; p=0.05)$ in newborns exposed to concentrations of endrin $\geq$ LD in utero, but a significantly lower odds of TSH $\geq 5 \mathrm{mU} / \mathrm{L}$ in newborns prenatally exposed to endosulfan-sulfate $\geq \mathrm{LD}$. A higher odds of TSH $\geq 5 \mathrm{mU} / \mathrm{L}(\mathrm{OR}=1.32$; $95 \% \mathrm{Cl}=0.95,1.83 ; p=0.09)$ was observed with higher $p, p^{\prime}$-DDE concentrations, although significance was not reached. With the exception of $\mathrm{HCB}$, which showed a negative and marginally significant association $(\beta=-0.15 ; 95 \% \mathrm{CI}=0.95,1.83 ; p=0.09$ ), no relationship was found between OCP concentrations in placenta samples and continuous TSH levels.

\section{Discussion}

The relationship between exposure to placental OCPs and TSH levels was investigated in 220 male newborns from the general population. It was found that the risk of cord blood TSH $\geq 5 \mathrm{mU} / \mathrm{L}$ was higher in neonates prenatally exposed to greater concentrations of endrin but lower in those exposed to greater concentrations of endosulfan-sulfate. Marginally significant associations were found between higher TSH concentrations and higher $p, p^{\prime}$-DDE and lower HCB. Exposure to OCPs during fetal development may exert some effects on thyroid status in neonates, as indicated in other studies

Table 4

Adjusted association of organochlorine pesticide (OCP) concentrations (ng/g placenta) with neonatal TSH levels (mU/L) measured in cord blood, INMA-Granada cohort, Southern Spain, 2000-2002 $(\mathrm{n}=220)^{\mathrm{a}}$.

\begin{tabular}{|c|c|c|c|c|c|c|}
\hline \multirow[t]{2}{*}{ OCPs } & \multicolumn{3}{|c|}{$\mathrm{TSH} \geq 5 \mathrm{mU} / \mathrm{L}^{\mathrm{b}}$} & \multicolumn{3}{|c|}{ Continuous $\mathrm{TSH}^{\mathrm{c}}$} \\
\hline & OR & $95 \% \mathrm{CI}$ & p-value & $\beta$ & $95 \%$ CI & p-value \\
\hline$o, p^{\prime}-\mathrm{DDT}^{\mathrm{d}}$ & 0.91 & $0.59 ; 1.42$ & 0.69 & 0.04 & $-0.07 ; 0.14$ & 0.49 \\
\hline$p, p^{\prime}-\mathrm{DDT}^{\mathrm{e}}$ & 0.79 & $0.39 ; 1.60$ & 0.51 & -1.0 & $-0.27 ; 0.07$ & 0.26 \\
\hline$p, p^{\prime}-\mathrm{DDE}^{\mathrm{d}}$ & 1.32 & $0.95 ; 1.83$ & 0.09 & 0.05 & $-0.26 ; 0.09$ & 0.26 \\
\hline$o, p^{\prime}-\mathrm{DDD}^{\mathrm{e}}$ & 0.77 & $0.37 ; 1.58$ & 0.48 & -0.09 & $-0.03 ; 0.12$ & 0.32 \\
\hline$\sum D_{D T s}{ }^{d}$ & 1.17 & $0.84 ; 1.63$ & 0.35 & -0.02 & $-0.10 ; 0.06$ & 0.57 \\
\hline $\mathrm{E}-\mathrm{I}^{\mathrm{d}}$ & 0.92 & $0.67 ; 1.25$ & 0.58 & -0.02 & $-0.10 ; 0.05$ & 0.53 \\
\hline E-II ${ }^{e}$ & 1.50 & $0.71 ; 3.17$ & 0.28 & -0.04 & $-0.22 ; 0.14$ & 0.68 \\
\hline E-diol ${ }^{\mathrm{d}}$ & 1.09 & $0.85 ; 1.40$ & 0.49 & 0.00 & $-0.05 ; 0.06$ & 0.89 \\
\hline E-ether ${ }^{\mathrm{d}}$ & 1.10 & $0.77 ; 1.56$ & 0.60 & -0.01 & $-0.10 ; 0.08$ & 0.80 \\
\hline E-sulfate & 0.36 & $0.17 ; 0.77$ & 0.008 & -0.12 & $-0.29 ; 0.05$ & 0.15 \\
\hline E-lactone $e^{e}$ & 0.80 & $0.38 ; 1.67$ & 0.55 & -0.05 & $-0.22 ; 0.12$ & 0.58 \\
\hline$\sum$ Endosulfans $^{\mathrm{d}}$ & 1.04 & $0.82 ; 1.31$ & 0.75 & -0.01 & $-0.06 ; 0.05$ & 0.84 \\
\hline Aldrin ${ }^{\mathrm{e}}$ & 0.94 & $0.43 ; 2.04$ & 0.87 & 0.02 & $-0.16 ; 0.21$ & 0.82 \\
\hline Endrin ${ }^{\mathrm{e}}$ & 2.05 & $1.01 ; 4.18$ & 0.05 & 0.03 & $-0.15 ; 0.20$ & 0.76 \\
\hline Dieldrin $^{\mathrm{e}}$ & 1.28 & $0.57 ; 2.86$ & 0.55 & -0.01 & $-0.21 ; 0.20$ & 0.93 \\
\hline Lindane $^{\mathrm{d}}$ & 0.92 & $0.59 ; 1.42$ & 0.71 & -0.03 & $-0.14 ; 0.07$ & 0.53 \\
\hline $\mathrm{HCB}^{\mathrm{e}}$ & 0.72 & $0.35 ; 1.47$ & 0.36 & -0.15 & $-0.31 ; 0.02$ & 0.09 \\
\hline Methoxychlor ${ }^{\mathrm{e}}$ & 1.72 & $0.81 ; 3.68$ & 0.16 & 0.04 & $-0.15 ; 0.22$ & 0.69 \\
\hline Mirex $^{\mathrm{e}}$ & 1.69 & $0.77 ; 3.69$ & 0.19 & 0.12 & $-0.07 ; 0.32$ & 0.22 \\
\hline
\end{tabular}

OR: Odds ratio; $\mathrm{CI}$ : confidence interval; $\beta$ : regression coefficient.

DDT: 1,1,1-trichloro-2,2-bis(4-chlorophenyl)-ethane; $\sum$ DDTs: sum of $o, p^{\prime}$-DDT, $p, p^{\prime}$-DDT, $p$, $p^{\prime}$-DDE, and $o, p^{\prime}$-DDD; E: endosulfan; $\sum$ Endosulfans: sum of E-I, E-II, E-diol, E-ether, E-sulfate, and E-lactone; HCB: hexachlorobenzene.

a Association of OCP concentrations with TSH levels, adjusted for maternal age, gestational age, smoking and alcohol consumption during pregnancy, parity, birth weight, maternal education, and placenta lipid concentration.

b Dichotomous TSH levels ( $\geq 5 \mathrm{mU} / \mathrm{L}$ ) as dependent variable.

c Log-transformed TSH levels as dependent variable.

d Concentrations transformed into natural logarithm.

e Categorical concentrations ( $\geq$ limit of detection). 
(Alvarez-Pedrerol et al., 2008; Asawasinsopon et al., 2006; LopezEspinosa et al., 2010; Ribas-Fitó et al., 2003); however, there was not a consistent pattern in the association shown in the present study.

Very little information is available on the specific thyroid effects of early exposure to most OCPs. Although TH concentrations have been consistently associated with PCBs and dioxins (Hagmar, 2003; Maervoet et al., 2007), evidence on the effect of OCPs on TH levels in vulnerable groups (e.g., pregnant women and infants) has been controversial (Alvarez-Pedrerol et al., 2008; Asawasinsopon et al., 2006; Dallaire et al., 2008; Maervoet et al., 2007; Takser et al., 2005). Previous findings in a Spanish community highly exposed to HCB showed no significant association between this pesticide and TSH concentrations at birth but, in agreement with our results, a close-tosignificant positive association between cord serum $p, p^{\prime}$-DDE and infant TSH levels $>10 \mathrm{mU} / \mathrm{L}$; the earlier study also reported a positive relationship with $\beta-\mathrm{HCH}$ (Ribas-Fitó et al., 2003). Two more recent Spanish studies found a positive association with $\beta-\mathrm{HCH}$ but not with HCB or DDT (Alvarez-Pedrerol et al., 2008; Lopez-Espinosa et al., 2010). Asawasinsopon et al. (2006) found no relation between cord blood TSH and maternal or cord serum levels of DDT, dieldrin, heptachlor, HCB or lindane, and there have been other reports of no association between TSH levels and OCP concentrations (Dallaire et al., 2008; Maervoet et al., 2007; Takser et al., 2005). Higher serum TSH levels were related to higher $p, p^{\prime}$-DDE exposure in Spanish pregnant women (Lopez-Espinosa et al., 2009), although TSH was not associated with maternal serum levels of DDTs and HCB (Chevrier et al., 2008). The present study found that neonatal TSH was related to placental endrin and endosulfan-sulfate. Limited data have been published on the effects of human exposure to these two pesticides on thyroid function (Dewailly et al., 1993). An unexpected finding was the negative relationship of TSH with endosulfan-sulfate and, although not significant, with $\mathrm{HCB}$, since a higher exposure to OCPs might be expected to increase TSH levels due to a decrease in free or total $\mathrm{T}_{4}$ neonatal levels (Maervoet et al., 2007; Takser et al., 2005).

Some mechanistic hypotheses can be proposed for the associations found in the present study. Thus, field and laboratory animal studies have demonstrated that DDT may be able to alter the production of THs and hormone availability at target tissues by competing with $\mathrm{T}_{4}$ for binding to purified human transthyretin (Scollon et al., 2004; Sormo et al., 2005; Verreault et al., 2004). Transthyretin is the main plasma $\mathrm{T}_{4}$ transporting protein in rats but may also play an important role in $\mathrm{T}_{4}$ transportation in the human brain. DDT has also been shown to disrupt THs by increasing TH peripheral metabolism through an induction of hepatic microsomal enzymes, resulting in an increased excretion of plasma $\mathrm{T}_{4}$ (Capen, 1994; Scollon et al., 2004). In contrast, an in vitro study found that neither $p, p^{\prime}$-DDT nor $p, p^{\prime}$-DDE interacted with transthyretin or thyroid-binding globulin (Cheek et al., 1999).

Few experimental data are available on the effects of endrin and endosulfan on the thyroid function. Interestingly, endrin was found to depress thyroid iodine uptake in various fish species, suggesting that it may compromise thyroid function by inhibiting TH synthesis and its intra-thyroidal storage, which can account for more than three months of TH reserves in humans (Bhattacharya et al., 1989; Singh and Singh, 1980). Evidence is emerging on the thyroid-disrupting effects of other pesticides, such as methoxychlor and endosulfan (Boas et al., 2006), but their mechanism of action has yet to be elucidated. Exposure to endosulfan was found to alter circulating $\mathrm{TH}$ levels in a study of fish, inhibiting $\mathrm{T}_{4}$ production/secretion and enhancing $\mathrm{T}_{4}$ clearance (Coimbra et al., 2005); low doses of endosulfan in the diet decreased plasma $\mathrm{T}_{4}$ levels, restored $\mathrm{T}_{3}$ levels, and depressed hepatic type I deiodinase activity at 21 days of exposure, but hepatic type III activity increased after 35 days of treatment. Contrary to expectations, a higher endosulfan dose did not alter TH plasma level or hepatic deiodinase activity in this study, suggesting that paradoxical results can be obtained depending on the dose and duration of exposure (Coimbra et al., 2005). Early exposure to endosulfan-sulfate could lead to significant alterations in $T_{4}$ feedback to the hypothalamus, resulting in decreasing TSH levels at birth, as it has been reported for placental and cord plasma PCBs (Sandau et al., 2002; Wang et al., 2005).

Humans may be exposed to mixtures of OCPs and numerous other compounds, hampering the prediction of effects on TH levels. In the present series, all placentas had measurable concentrations of at least one of the 17 OCPs investigated, reflecting the ubiquity of exposure in the population, as previously reported (Lopez-Espinosa, 2006; LopezEspinosa et al., 2007). Chemicals may have different effects on the thyroid axis or act synergistically (Crofton et al., 2005). Therefore, alterations in TSH levels might result not only from prenatal exposure to endrin or endosulfan-sulfate but also from exposure to other OCPs, alone or in combination. It should be borne in mind that the INMAGranada birth cohort is entirely composed of male infants, whose hormonal levels might have differed from those of females. Thus, Wang et al. (2005) described a gender difference in the sensitivity to EDs, reporting that prenatal exposure to dioxins - which have antiestrogenic activity - increased neonatal $\mathrm{T}_{4}$ levels in female but not male infants.

A Spanish study of another INMA cohort (Alvarez-Pedrerol et al., 2007) showed that THs were related to cognitive function and attention behavior among preschoolers, suggesting that even small changes in TH levels may have a significant effect on brain function. Concentrations of DDT in cord serum were associated with a decrease in cognitive skills at preschool age in two other INMA cohorts (RibasFitó et al., 2006). The present cohort is being followed up throughout childhood, and a recent longitudinal study showed that prenatal exposure to mirex (in 104 of these children) had a negative effect on cognitive development at the age of 4 years (Puertas et al., 2010). Although mirex did not show a significant effect on neonatal TSH status in the present study, a positive trend $(p=0.07)$ was found with continuous TSH in the crude analysis and with TSH $\geq 5 \mathrm{mU} / \mathrm{L}$ $(\mathrm{OR}=1.69 ; p=0.19)$ in the adjusted analysis. A further study has been recently conducted by our group into the impact of neonatal TSH levels on neurodevelopment at the age of 4 years (Freire et al., 2010b), which will be also investigated in the same cohort at the age of 910 years.

Other study strengths are that we examined the effects of a large number of covariates identified from the literature. Neonatal TSH levels were assessed using the same technique as in other Spanish studies (part of routine early screening for hypothyroidism in Spanish hospitals), supporting the validity of the present study. We measured prenatal exposure to OCPs in placenta samples, hampering comparison of our findings with previous reports on this issue. Nevertheless, a high correlation has been demonstrated among OC concentrations in maternal serum, cord serum and placenta (Bergonzi et al., 2009; Wang et al., 2004), and placental analysis offers the opportunity to measure the exposure of both mother and fetus to EDs without requiring an invasive procedure. A limitation of our study is that only concentrations of TSH were determined, and no data were available on total or free $T_{4} / T_{3}$. However, TSH is considered a more sensitive indicator of thyroid disruption in comparison to $\mathrm{T}_{4}$ or $\mathrm{T}_{3}$ levels (The National Academy of Clinical Biochemistry, 2007).

\section{Conclusions}

This is the first report on the relationship between TSH status at birth and prenatal exposure to a wide range of OCPs as measured in the placenta. Although some associations were detected between OCP exposure and TSH cord blood levels, no clear pattern emerged on the influence of the compounds measured in placenta tissue on neonatal $\mathrm{TH}$ status. The impact of environmental chemicals on thyroid hormone levels and metabolism is a matter of growing concern, and further research is warranted to elucidate the causal 
relationship between endocrine-disrupting chemicals and thyroid hormones.

\section{Funding sources}

This work was supported by the Consejería de Salud de la Junta de Andalucía [grant number P09-CTS-5488]; the Spanish Ministry of Health [grant number FIS07/0252]; and the European Commission [grant number CONTAMED-FP7-ENV-2007-1-212502 and EUS200803574].

\section{Acknowledgments}

The authors are grateful to Richard Davies for editorial assistance. The results would not have been achieved without the selfless collaboration of the study participants, the staff at the Department of Gynecology and Pediatrics of the San Cecilio University Hospital and at the Environmental Health Department of the Consejería de Salud de la Junta de Andalucía.

\section{References}

Alvarez-Pedrerol M, Ribas-Fitó N, Torrent M, Julvez J, Ferrer C, Sunyer J. TSH concentration within the normal range is associated with cognitive function and ADHD symptoms in healthy preschoolers. Clin Endocrinol 2007;66:890-8.

Alvarez-Pedrerol M, Ribas-Fitó N, Torrent M, Carrizo D, Garcia-Esteban R, Grimalt JO et al. Thyroid disruption at birth due to prenatal exposure to beta-hexachlorocyclohexane. Environ Int 2008;34:737-40.

Alvarez-Pedrerol M, Guxens M, Ibarluzea J, Rebagliato M, Rodriguez A. Espada M, et al. Organochlorine compounds, iodine intake, and thyroid hormone levels during pregnancy. Environ Sci Technol 2009a;43:7909-15.

Alvarez-Pedrerol M, Guxens M, Mendez M, Canet Y, Martorell R, Espada M, et al. Iodine levels and thyroid hormones in healthy pregnant women and birth weight of their offspring. Eur J Endocrinol 2009b;160:423-9.

Asawasinsopon R, Prapamontol T, Prakobvitayakit O, Vaneesorn Y, Mangklabruks A Hock B. The association between organochlorine and thyroid hormone levels in cord serum: a study from northern Thailand. Environ Int 2006;32:554-9.

ATSDR. Toxicological profile for DDT, DDE and DDD. Atlanta, GA: Agency for Toxic Substances and Disease Registry; 2002a.

ATSDR. Toxicological profile for hexachlorobenzene. Atlanta, GA: Agency for Toxic Substances and Disease Registry; 2002b.

Beard J. DDT and human health (Australian Rural Health Research Collaboration). Sci Total Environ 2006:344:78-89.

Bergonzi R, Specchia C, Dinolfo M, Tomasi C, De Palma G, Frusca T, et al. Distribution of persistent organochlorine pollutants in maternal and foetal tissues: data from an Italian polluted urban area. Chemosphere 2009;76:747-54.

Bhattacharya T, Bhattacharya S, Ray AK, Dey S. Influence of industrial pollutants on thyroid function in Channa punctatus (Bloch). Indian J Exp Biol 1989;27:65-8.

Boas M, Feldt-Rasmussen U, Skakkebaek NE, Main KM. Environmental chemicals and thyroid function. Eur J Endocrinol 2006;154:599-611.

Capen CC. Mechanisms of chemical injury of thyroid gland. Prog Clin Biol Res 1994;387: 173-91.

Cerrillo I, Granada A, Lopez-Espinosa MJ, Olmos B, Jiménez M, Caño A, et al. Endosulfan and its metabolites in fertile women, placenta, cord blood, and human milk. Environ Res 2005;98:233-9.

Coimbra AM, Reis-Henriques MA, Darras VM. Circulating thyroid hormone levels and iodothyronine deiodinase activities in Nile tilapia (Oreochromis niloticus) following dietary exposure to Endosulfan and Aroclor 1254. Comp Biochem Physiol C Toxicol Pharmacol 2005;141:8-14.

Cortés Castell E, Chamorro Ureña F, Dulín Iñíguez E, Eguileor Gurtubai I, Espada SáenzTorre M, Remón Alvarez-Arenas J, et al. On behalf of the Comisión de Errores Metabólicos Congénitos de la Sociedad Española de Bioquímica Clínica y Patología Molecular (SEQC), 2002. Neonatal screening and collaboration among scientific institutions. An Esp Pediatr 2002;56:201-3.

Crofton KM, Craft ES, Hedge JM, Gennings C, Simmons JE, Carchman RA, et al. Thyroidhormonedisrupting chemicals: evidence for dose-dependent additivity or synergism. Environ Health Perspect 2005;113:1549-54.

Cheek AO, Kow K, Chen J, McLachlan JA. Potential mechanisms of thyroid disruption in humans: interaction of organochlorine compounds with thyroid receptor, transthyretin, and thyroid-binding globulin. Environ Health Perspect 1999;107: 273-8.

Chevrier J, Eskenazi B, Holland N, Bradman A, Barr DB. Effects of exposure to polychlorinated biphenyls and organochlorine pesticides on thyroid function during pregnancy. Am J Epidemiol 2008;168:298-310.

Dallaire R, Dewailly E, Ayotte P, Muckle G, Laliberté C, Bruneau S. Effects of prenatal exposure to organochlorines on thyroid hormone status in newborns from two remote coastal regions in Québec, Canada. Environ Res 2008;108:387-92.
DeCastro BR, Korrick SA, Spengler JD, Soto AM. Estrogenic activity of polychlorinated biphenyls present in human tissue and the environment. Environ Sci Technol 2006:40:2819-25.

Dewailly E, Bruneau S, Ayotte P, Laliberte C, Gingras S, Belanger D, et al. Health status of birth of Inuit newborn prenatally exposed to organochlorines. Chemosphere 1993;27:359-66.

Endosulfan Preliminary Dossier. Umweltbundesamt, Berlin, p. 55; 2003.

Fernández MF, Rivas A, Olea-Serrano F, Cerrillo I, Molina-Molina JM, Araque P, Martínez-Vidal JL, Olea N. Assessment of total effective xenoestrogen burden in adipose tissue and identification of chemicals responsible for the combined estrogenic effect. Anal Bioanal Chem 2004;379:163-70.

Fernández MF, Olmos B, Granada A, Lopez-Espinosa MJ, Molina-Molina JM, Fernández $\mathrm{JM}$, et al. Human exposure to endocrine disrupting chemicals and prenatal risk factors for cryptorchidism and hypospadias. A nested case-control study. Environ Health Perspect 2007;15:8-14.

Freire C, Ramos R, Puertas R, Lopez-Espinosa MJ, Julvez J, Aguilera I, et al. Association of traffic-related air pollution with cognitive development in children. J Epidemio Community Health 2010a;64:223-8.

Freire C, Ramos R, Amaya E, Fernández MF, Santiago-Fernández P, Lopez-Espinosa MJ et al. Newborn TSH concentration and its association with cognitive development in healthy boys. Eur J Endocrinol 2010b;163:901-9.

Hagmar L. Polychlorinated biphenyls and thyroid status in humans: a review. Thyroid 2003; 13:1021-8.

Langer P, Tajtaková M, Kocan A, Vlcek M, Petrik J, Chovancova J, et al. Multiple organochlorine pollution and the thyroid. Endocr Regul 2006;40:46-52.

Lopez-Espinosa MJ. Mother-infant exposure to environmental chemical compounds with hormonal activity via the placenta [Doctoral Thesis]. Granada: Universidad de Granada; 2006.

Lopez-Espinosa MJ, Granada A, Carreño J, Salvatierra MT, Olea-Serrano F, Olea N Organolorine pesticides in placentas from Southern Spain and some related factors. Placenta 2007;28:631-8.

Lopez-Espinosa MJ, Vizcaino E, Murcia M, Llop S, Espada M, Seco V, et al. Association between thyroid hormone levels and 4,4'-DDE concentrations in pregnant women (Valencia, Spain). Environ Res 2009;109:479-85.

Lopez-Espinosa MJ, Vizcaino E, Murcia M, Fuentes V, Garcia AM, Rebagliato M, et al. Prenatal exposure to organochlorine compounds and neonatal thyroid stimulating hormone levels. J Expo Sci Environ Epidemiol 2010;20:579-88.

Maervoet J, Vermeir G, Covaci A, Nelen V, Baeyens W, Schepens P, et al. Association of thyroid hormone concentrations with levels of organochlorine compounds in cord blood of neonates. Environ Health Perspect 2007;115:1780-6.

PNA Convenio Estocolmo y Reglamento 850/2004.. Plan nacional de aplicación del convenio de estocolmo y el reglamento $850 / 2004$, sobre contaminantes orgánicos persistentes. Subdirección general de calidad del aire y prevención de riesgos del ministerio de medio ambiente; 2007.

Puertas R, Lopez-Espinosa MJ, Cruz F, Ramos R, Freire C, Pérez-García M, et al. Prenatal exposure to mirex impairs neurodevelopment at age of 4 years. Neurotoxicology 2010;31:154-60.

Ribas-Fitó N, Sala M, Cardo E, Mazon C, De Muga ME, Verdú A, et al. Organochlorine compounds and concentrations of thyroid stimulating hormone in newborns. Occup Environ Med 2003;60:301-3.

Ribas-Fitó N, Torrent M, Carrizo D, Muñoz-Ortiz L, Julvez J, Grimalt JO, et al. In utero exposure to background concentrations of DDT and cognitive functioning among preschoolers. Am J Epidemiol 2006;164:955-62.

Ribas-Fitó N, Torrent M, Carrizo D, Júlvez J, Grimalt JO, Sunyer J. Exposure to hexachlorobenzene during pregnancy and children's social behavior at 4 years of age. Environ Health Perspect 2007;115:447-50.

Sandau CD, Ayotte P, Dewailly E, Duffe J, Norstrom RJ. Pentachlorophenol and hydroxylated polychlorinated biphenyl metabolites in umbilical cord plasma of neonates from coastal populations in Quebec. Environ Health Perspect 2002;110: 411-7.

Singh H, Singh TP. Thyroid activity and TSH potency of the pituitary gland and blood serum in response to cythion and hexadrin treatment in the freshwater catfish Heteropneustes fossilis (Blosh). Environ Res 1980;22:184-9.

Scollon EJ, Carr JA, Cobb GP. The effect of flight, fasting and p, p'-DDT on thyroid hormones and corticosterone in Gambel's white-crowned sparrow, Zonotrichia leucophrys gambelli. Comp Biochem Physiol C Toxicol Pharmacol 2004;137:179-89.

Schisterman EF, Whitcomb BW, Buck Louis GM, Louis TA. Lipid adjustment in the analysis of environmental contaminants and human health risks. Environ Health Perspect 2005;113:853-7.

Sormo EG, Jussi I, Jussi M, Braathen M, Skaare JU, Jenssen BM. Thyroid hormone status in gray seal (Halichoerus grypus) pups from the Baltic Sea and the Atlantic Ocean in relation to organochlorine pollutants. Environ Toxicol Chem 2005;24:610-6.

Takser L, Mergler D, Baldwin M, Smargiassi A, Lafond J. Thyroid hormones in pregnancy in relation to environmental exposure to organochlorine compounds and mercury. Environ Health Perspect 2005;113:1039-45.

The National Academy of Clinical Biochemistry. Guía de consenso para el diagnóstico y seguimiento de la enfermedad tiroidea. Acta Bioquímica Clínica Latinoamericana 2007;41:87-119.

Toft G, Hagmar L, Giwercman A, Bonde JP. Epidemiological evidence on reproductive effects of persistent organochlorines in humans. Reprod Toxicol 2004;19:5-26.

Verreault J, Skaare JU, Jenssen BM. Effects of organochlorine contaminants on thyroid hormone levels in Arctic breeding glaucous gulls, Larus hyperboreus. Environ Health Perspect 2004;112:532-7.

Wang SL, Lin CY, Guo YL, Lin LY, Chou WL, Chang LW. Infant exposure to polychlorinated dibenzo-p-dioxins, dibenzofurans and biphenyls (PCDD/Fs, PCBs)-correlation between prenatal and postnatal exposure. Chemosphere 2004;54:1459-73. 
Wang SL, Su PH, Jong SB, Guo YL, Chou WL, Papke O. In utero exposure to dioxins and polychlorinated biphenyls and its relations to thyroid function and growth hormone in newborns. Environ Health Perspect 2005;113:1645-50.

Williams GR. Neurodevelopmental and neuropsychological actions of thyroid hormone.

J Neuroendocrinol 2008;20:784-94.
World Health Organization (WHO). Assessment of iodine deficiency disorders and monitoring their elimination: a guide for programme managers3rd ed. 2007.

Zoeller T. Environmental chemicals impacting the thyroid: targets and consequences. Thyroid 2007;17:811-7. 